\title{
Editorial: The Effect of Carbohydrate Restriction on Cancer and Metabolic Syndrome
}

\author{
Ingrid Elisia $^{1 *}$, Sylvia Santosa ${ }^{2}$, David G. Popovich ${ }^{3}$ and Gerald Krystal ${ }^{1}$ \\ ${ }^{1}$ Terry Fox Laboratory, British Columbia Cancer Research Centre, Vancouver, BC, Canada, ${ }^{2}$ Department of Health, \\ Kinesiology and Applied Physiology, Concordia University, Montreal, QC, Canada, ${ }^{3}$ School of Food and Advanced \\ Technology, Massey University, Palmerston North, New Zealand
}

Keywords: cancer, sugar, ketogenic, low carbohydrate diet, metabolic syndrome

\section{Editorial on the Research Topic}

\section{The Effect of Carbohydrate Restriction on Cancer and Metabolic Syndrome}

OPEN ACCESS

Edited by:

Clelia Madeddu,

University of Cagliari, Italy

Reviewed by:

Richard David Feinman,

SUNY Downstate Medical Center,

United States

*Correspondence:

Ingrid Elisia

ielisia@bccrc.ca

Specialty section: This article was submitted to

Clinical Nutrition,

a section of the journal

Frontiers in Nutrition

Received: 27 November 2021

Accepted: 24 January 2022

Published: 14 February 2022

Citation:

Elisia I, Santosa S, Popovich DG and Krystal G (2022) Editorial: The Effect of

Carbohydrate Restriction on Cancer and Metabolic Syndrome.

Front. Nutr. 9:823302.

doi: 10.3389/fnut.2022.823302
Currently, low carbohydrate (LC) diets, including ketogenic diets (KDs), are not only gaining popularity for weight loss but are also being investigated for the prevention and treatment of cancer, diabetes, and metabolic syndrome (1-3). In this special issue, we present six articles evaluating the safety/efficacy of $\mathrm{LC} / \mathrm{KD}$ in the prevention and treatment of cancer and metabolic syndrome.

Using glucose restriction to prevent and treat cancer is based on the observation that cancer cells typically consume and require more glucose than normal cells (the Warburg effect) (4). Currently, data supporting the safety and efficacy of a $\mathrm{LC} / \mathrm{KD}$ for the prevention/treatment of cancer are limited to pre-clinical animal studies and a few case reports. Herein, we present a systematic review and meta-analysis of animal studies by Li et al. which point to an overall anti-tumor effect of KDs, albeit with a limited number of tumor types. However, there is a lack of high-quality data in humans because no randomized placebo-controlled double-blind clinical trials have been carried out in this area. Nevertheless, the online survey results in the Tulipan and Kofler article suggest that LC diets improve the quality of life of cancer patients and normalize their body weight. The survey results also indicate that some cancer patients adopt a KD or LC diet on their own without professional counseling and without informing their doctors.

Sukkar and Muscaritoli discuss the various dietary regimens being used currently by cancer patients and the scientific rationale for their use. They stress the importance of cancer patients receiving medical supervision when adopting a LC diet since these diets may affect their wellbeing during cancer treatment, particularly since there are many types of LC diets. They find that some types of LC diets may lead to vitamin/mineral deficiencies and unwanted weight loss that may be detrimental for cancer patients. Since clinical evidence demonstrating the safety and efficacy of LC diets as an adjunct for cancer patients is currently lacking, there is a gap between the advice that can be given by medical/health care workers and the professional advice that cancer patients need. Related to this, Haskins et al. provide a thorough review of traditional cancer therapies and possible synergies with LC diets. Given that there are many ongoing clinical trials investigating the safety and efficacy of LC/KDs in combination with various cancer therapies, this gap could be filled in the not too distant future. 
The main mechanism by which a $\mathrm{KD}$ is thought to elicit clinical benefits is by inducing a metabolic switch that pushes the body to utilize fats via ketogenesis rather than glucose as an energy source. During ketogenesis, fats are broken down to generate ketone bodies, which can then fuel oxidative phosphorylation in normal cells for ATP generation. Cancer cells are thought to lack one or more of the ketolytic enzymes necessary to break down ketone bodies for energy, thus leading to their selective death when patients are on a KD. The observation that the health benefits of a KD typically correlate with an increase in ketone bodies has given rise to the use of ketone body supplementation alone to evoke a comparable clinical impact as a KD. However, Buga et al. suggest that while a KD favorably impacts body composition, ketone salts themselves have no impact, suggesting the importance of a whole body metabolic switch and not just the elevation of ketone bodies in producing health benefits.

Lastly, Elisia and Krystal highlight the fact that many KDs are high in saturated fats, known to be pro-inflammatory (57). Thus, some KDs may contribute to chronic inflammation and, in turn, cancer development (8). Other fats, like unsaturated omega 3 fatty acids have anti-inflammatory properties $(5,9)$, and so may reduce cancer development. The authors therefore encourage future studies to indicate the types of fats used. Also, since not all cancers rely on glucose [e.g., early stage prostate cancer appears to prefer fatty acids as a nutrient source (10)], a LC/KD may also not be effective for all types of cancer. Elisia and Krystal also report that the short-chain fatty acids (SCFAs) generated from the fermentation of soluble fiber and

\section{REFERENCES}

1. Hyde PN, Sapper TN, Crabtree CD, LaFountain RA, Bowling ML, Buga A, et al. Dietary carbohydrate restriction improves metabolic syndrome independent of weight loss. JCI insight. (2019) 4:e128308. doi: 10.1172/jci.insight.128308

2. Feinman RD, Pogozelski WK, Astrup A, Bernstein RK, Fine EJ, Westman EC, et al. Dietary carbohydrate restriction as the first approach in diabetes management: critical review and evidence base. Nutrition. (2015) 31:113. doi: 10.1016/j.nut.2014.06.011

3. Fine EJ, Segal-Isaacson CJ, Feinman RD, Herszkopf S, Romano MC, Tomuta $\mathrm{N}$, et al. Targeting insulin inhibition as a metabolic therapy in advanced cancer: a pilot safety and feasibility dietary trial in 10 patients. Nutrition. (2012) 28:1028-35. doi: 10.1016/j.nut.2012.05.001

4. Vander Heiden MG, Cantley LC, Thompson CB. Understanding the Warburg effect: the metabolic requirements of cell proliferation. Science. (2009) 324:1029-33. doi: 10.1126/science.1160809

5. Shi H, Kokoeva MV, Inouye K, Tzameli I, Yin H, Flier JS. TLR4 links innate immunity and fatty acid-induced insulin resistance. J Clin Invest. (2006) 116:3015-25. doi: 10.1172/JCI28898

6. Cani PD, Amar J, Iglesias MA, Poggi M, Knauf C, Bastelica D, et al. Metabolic endotoxemia initiates obesity and insulin resistance. Diabetes. (2007) 56:176172. doi: 10.2337/db06-1491

7. Fritsche KL. The science of fatty acids and inflammation. Adv Nutr. (2015) 6:293S-301S. doi: 10.3945/an.114.006940

8. Coussens LM, Werb Z. Inflammation and cancer. Nature. (2002) 420:8607. doi: $10.1038 /$ nature 01322 resistant starch by the gut microbiome are more potently antiinflammatory than beta-hydroxybutyrate-an important ketone body produced when one adopts a LC/KD. They suggest that one of the limitations of a $\mathrm{LC} / \mathrm{KD}$ is that it is typically deficient in soluble fiber/resistant starch and thus results in low levels of anti-inflammatory SCFAs. Whether a LC/KD can be optimized so that soluble fiber/resistant starch can be incorporated is another research question warranting further investigation.

To conclude, while LC/KDs have given promising results to date, there is still more research required before a medical professional can advise whether a LC/KD may offer health benefits in the treatment/prevention of specific cancers and/or metabolic syndrome. Considering that there is a clear interest in $\mathrm{LC} / \mathrm{KD}$ s for metabolic syndrome and as a cancer adjunct, future research should aim to provide evidence of efficacy against tumors with different metabolic requirements as well as define the role of different fat types in LC/KDs on inflammatory status.

\section{AUTHOR CONTRIBUTIONS}

IE wrote the first draft. SS, DP, and GK edited and approved the final draft. All authors contributed to the article and approved the submitted version.

\section{FUNDING}

The research was supported by the Lotte and John Hecht Memorial Foundation (\#4340), with core support from the BC Cancer Foundation and BC Cancer.

9. Calder PC. Omega-3 polyunsaturated fatty acids and inflammatory processes: nutrition or pharmacology? Br J Clin Pharmacol. (2013) 75:64562. doi: 10.1111/j.1365-2125.2012.04374.x

10. Butler LM, Centenera MM, Swinnen JV. Androgen control of lipid metabolism in prostate cancer: novel insights and future applications. Endocr Relat Cancer. (2016) 23:R219-27. doi: 10.1530/ERC15-0556

Conflict of Interest: The authors declare that the research was conducted in the absence of any commercial or financial relationships that could be construed as a potential conflict of interest.

Publisher's Note: All claims expressed in this article are solely those of the authors and do not necessarily represent those of their affiliated organizations, or those of the publisher, the editors and the reviewers. Any product that may be evaluated in this article, or claim that may be made by its manufacturer, is not guaranteed or endorsed by the publisher.

Copyright (C) 2022 Elisia, Santosa, Popovich and Krystal. This is an open-access article distributed under the terms of the Creative Commons Attribution License (CC $B Y)$. The use, distribution or reproduction in other forums is permitted, provided the original author(s) and the copyright owner(s) are credited and that the original publication in this journal is cited, in accordance with accepted academic practice. No use, distribution or reproduction is permitted which does not comply with these terms. 